\title{
Mechanical stabilization of the Levitron's realistic model
}

\author{
Arturo Olvera ${ }^{1, a}$, Abraham De la Rosa ${ }^{1}$, and Claudia M Giordano ${ }^{2}$ \\ 1 IIMAS-UNAM 04510 Mexico, D.F. Mexico \\ 2 Instituto de Astrofsica de La Plata, Facultad de Ciencias Astronomicas \\ y Geofsicas - UNLP Paseo del Bosque, B1900FWA La Plata, Argentina
}

Received 15 January 2016 / Received in final form 6 April 2016

Published online XX XX 2016

\begin{abstract}
The stability of the magnetic levitation showed by the Levitron was studied by M.V. Berry as a six degrees of freedom Hamiltonian system using an adiabatic approximation. Further, H.R. Dullin found critical spin rate bounds where the levitation persist and R.F. Gans et al. offered numerical results regarding the initial conditions' manifold where this occurs. In the line of this series of works, first, we extend the equations of motion to include dissipation for a more realistic model, and then introduce a mechanical forcing to inject energy into the system in order to prevent the Levitron from falling. A systematic study of the flying time as a function of the forcing parameters is carried out which yields detailed bifurcation diagrams showing an Arnold's tongues structure. The stability of these solutions were studied with the help of a novel method to compute the maximum Lyapunov exponent called MEGNO. The bifurcation diagrams for MEGNO reproduce the same Arnold's tongue structure.
\end{abstract}

\section{Introduction}

The Levitron is a mechanical device conformed basically by two parts: the top, a magnetized rotationally symmetric rigid body of uniform mass that behaves as a magnetic dipole, and the base that provides a permanent magnetic field such that the interaction with the magnetic dipole compensates the gravitational force acting on the top when put to spin over it.

Magnetic levitation of spinning bodies was discovered by Roy Harrigan who developed a device using a square magnetic base and by calibrating the volume and mass of a magnetic top he achieved the inertial and magnetic momenta leading to its persistent magnetic levitation. The Earnshaw's theorem [1] establishes the rules for the magnetic levitation of static dipoles, where no stable configuration of static dipoles is possible, leaving the Levitron's dynamics to be understood.

a e-mail: aoc@mym.iimas.unam.mx 


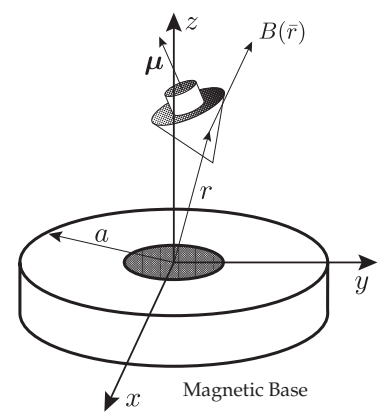

Fig. 1. Diagram of the Levitron ${ }^{\mathrm{TM}}: B(\bar{r})$ is the static magnetic field of the base and $\boldsymbol{\mu}$ is the top magnetic dipole

Many different attempts to comprehend how the Levitron's mechanism works have been done. In fact, B. Hones in 1995 was among the firsts to address the study of the Levitron; however, his discussion did not allow for levitation over a circular magnetic base which is indeed possible. The more theoretical approaches to explain the dynamics of this device were made principally by [2] and [3] in 1996. A Hamiltonian formulation of six degrees of freedom and an adiabatic theory to prove that the magnetic levitation is possible only for some ranges of the spinning rates is given in [2]. Therein the role of the precession movement of the top to allow persisting levitation is also shown. In [3] both physical and numerical experiments were performed to confirm the theoretical results of M.V. Berry. Later on, in [4], the Hamiltonian approach was further developed by studying the local behavior near an equilibrium point of the system, to find invariant regions in the phase space where persistent levitation of the top is feasible.

The results presented in [4] are very useful for the numerical analysis of the equations. Also, in [5] dimensionless constants that facilitate the the analysis of simulations are introduced. Both papers follow the guideline drew by [2] and are the base for the present work. Nevertheless none of the cited papers have studied the Levitron's dynamics considering energy losses caused by friction, in [6] we can find a first attempt to introduce losses in the dynamic of the Levitron.

The aim of this effort is to present a numerical study of the Levitron's dynamics. For the sake of completeness and clarity the equations of motion are included, and invariant regions in phase space as well as equilibrium states are described. A numerical verification of the range for the spinning rates given in [2] is performed, a persistent levitation of the top being observed. Then, dissipative terms are introduced in the equations of motion to model the friction with the air in a more realistic setting resulting finite flight times. Since the system with dissipative terms is no longer Hamiltonian, a strategy to inject energy into the system is proposed, which will be numerically explored studying the possibility to counteract the effect of dissipation preventing the top to fall down. It should be pointed out that this approach is of a mechanical nature, that is, no electromagnetic drive [6] or air jet propulsion [3] are considered. This scheme corresponds to a mechanical perturbation of the position of the magnetic base using a parametric excitation.

Furthermore a proper method for computing the Lyapunov exponents is chosen to study the stability of the solutions of the more complex equations of motion, including both dissipation and perturbation terms. These equations are numerically studied over a grid in the perturbation parameter space to determine the regions where maximum times of flight are observed. In a similar way the stability of the numerical solutions are also studied. The final section is devoted to present the conclusions of the overall analysis. 


\section{Equations of motion}

75 In order to obtain the equations of motion of the Levitron, we follow the scheme 7 delivered in [5] where the magnetic base is modelled as a ring dipole of radius $a$, the 77 magnetic potential along the vertical axis being

$$
V_{0}(z)=\frac{M}{2} \frac{z a}{\left(a^{2}+z^{2}\right)^{3 / 2}}
$$

The magnetic field $B(\bar{r})=-\nabla V(\bar{r})$ is defined by the magnetic potential

$$
V(\bar{r})=\Phi_{0}-\frac{\rho^{2}}{4} \Phi_{2}(z) \pm \cdots, \quad \text { where } \quad \Phi_{k}(z)=\frac{\mathrm{d}^{k}}{\mathrm{~d} z^{k}} V_{0}(z)
$$

Finally, the potential energy of the Levitron is given by the gravitational component and the magnetic interaction of the top with the magnetic field (see [7] for details)

$$
\Psi(\bar{r}, \mathbf{R})=m g z-\langle B(\bar{r}), \boldsymbol{\mu}\rangle
$$

where $\boldsymbol{\mu}=\mu \mathbf{R} e_{z}$ is the magnetic momentum of the Levitron in the external reference frame, $\mathbf{R}$ being a rotational matrix.

Meantime, the kinetic energy is given by the model of the eulerian top (see [8] and $[5])$.

For the sake of convenience we will deal with the dimensionless equations of motion of the spinning top, by scaling by the length $a$ (the radius of the dipole ring) and a consistent time scale $\tau$. Therefore we define

$$
x=a X, \quad y=a Y, \quad z=a Z, \quad t=(a / g)^{1 / 2} \tau .
$$

By (2) and (1), $V$ can be written in terms of the dimensionless variables as

$$
V=\frac{M_{e}}{4 \pi a^{2}}\left[f_{0}(Z)+\left(X^{2}+Y^{2}\right) f_{2}(Z)+O\left(\left(X^{2}+Y^{2}\right)^{2}\right)\right]=: \frac{M_{e}}{4 \pi a^{2}} \Phi,
$$

86 with

$$
f_{0}(Z)=\frac{Z}{\left(1+Z^{2}\right)^{3 / 2}}, \quad f_{2}(Z)=-\frac{3}{4} \frac{\left(2 Z^{2}-3\right) Z}{\left(1+Z^{2}\right)^{7 / 2}}
$$

where $M_{e}=2 \pi M a$ is the net strength of the dipoles of the ring and $a$ is the effective radius.

The Hamiltonian proposed in [5] is

$$
\begin{aligned}
\mathcal{H}= & \frac{1}{2}\left(p_{X}^{2}+p_{Y}^{2}+p_{Z}^{2}+\frac{p_{\theta}^{2}}{A}+\frac{\left[p_{\psi}-p_{\phi} \cos \theta\right]^{2}}{A \sin ^{2} \theta}+\frac{p_{\phi}^{2}}{C}\right) \\
& -\mathcal{M}\left[\sin \theta\left(\cos \psi \frac{\partial \Phi}{\partial X}+\sin \psi \frac{\partial \Phi}{\partial Y}\right)+\cos \theta \frac{\partial \Phi}{\partial Z}\right]+Z,
\end{aligned}
$$


and the concomitant equations of motion read

$$
\begin{aligned}
\dot{X} & =p_{X} \\
\dot{Y} & =p_{Y} \\
\dot{Z}= & p_{Z} \\
\dot{\theta}= & \frac{p_{\theta}}{A} \\
\dot{\psi}= & \frac{p_{\psi}-p_{\phi} \cos \theta}{A \sin ^{2} \theta} \\
\dot{\phi}= & -\cos \theta \cdot \frac{p_{\psi}-p_{\phi} \cos \theta}{A \sin ^{2} \theta}+\frac{p_{\phi}}{C} \\
p_{X}= & 2 \mathcal{M}\left[f_{2}(Z) \sin \theta \cos \psi+X f_{2}^{\prime}(Z) \cos \theta\right] \\
p_{Y}= & 2 \mathcal{M}\left[f_{2}(Z) \sin \theta \sin \psi+Y f_{2}^{\prime}(Z) \cos \theta\right] \\
p_{Z}= & \mathcal{M}\left[2 f_{2}^{\prime}(Z) \sin \theta(X \cos \psi+Y \sin \psi)+\cos \theta\left(f_{0}^{\prime \prime}(Z)+\left(X^{2}+Y^{2}\right) f_{2}^{\prime \prime}(Z)\right)\right] \\
\dot{p_{\theta}}= & -\frac{\left(p_{\phi} \cos \theta-p_{\psi}\right)\left(p_{\psi} \cos \theta-p_{\phi}\right)}{A \sin 3} \\
& +\mathcal{M}\left[2 f_{2}(Z) \cos \theta(X \cos \psi+Y \sin \psi)-\sin \theta\left(f_{0}^{\prime}(Z)+\left(X^{2}+Y^{2}\right) f_{2}^{\prime}(Z)\right)\right] \\
\dot{p_{\psi}}= & 2 \mathcal{M} f_{2}(Z) \sin \theta(Y \cos \psi-X \sin \psi) \\
\dot{p_{\phi}}= & 0
\end{aligned}
$$

$91 \quad$ where

$$
A=\frac{\Theta_{1}}{m a^{2}}, \quad C=\frac{\Theta_{2}}{m a^{2}}, \quad \mathcal{M}=\frac{-\mu M_{e}}{4 \pi m g a^{4}},
$$

are positive constants, $\Theta_{1}$ and $\Theta_{2}$ are the inertial moments of the top, $m$ is the mass and $M_{e}$ is the net strengh of the dipoles of the ring. Of course $\mathcal{H}$ is a conserved quantity as well as $p_{\phi}$.

We want to remark that the kynetic energy of our hamiltonian function $\mathcal{H}$ is defined with the eulerian angular coordinates such that $\theta=0$ is a singular value. In $[6,9]$ and [4], they use a different set of angular coordinates where $\theta=0$ is now a regular value of the kynetic energy.

Following the ideas in [4], it can be seen that

$$
\text { Inv }=\left\{X=Y=0, \theta=0, p_{X}=p_{Y}=0, p_{\theta}=0, p_{\psi}=p_{\phi}\right\}
$$

is an invariant set of the equations of motion. The corresponding Hamiltonian is an is

$$
\mathcal{H}_{\mathrm{Inv}}=\frac{1}{2}\left(p_{Z}^{2}+\frac{p_{\phi}^{2}}{C}\right)-\mathcal{M}\left[\frac{\partial \Phi}{\partial Z}\right]+Z
$$


The dynamics in this region is given by

$$
\begin{array}{ll}
\dot{Z}=p_{Z} & \dot{p_{Z}}=\mathcal{M} f_{0}^{\prime \prime}(Z)-1 \\
\dot{\phi}=\frac{p_{\phi}}{C}=\sigma & \dot{p_{\phi}}=0
\end{array}
$$

This system has two fixed points which are defined by the relationship

$$
f_{0}^{\prime \prime}(Z)=\frac{1}{\mathcal{M}} .
$$

In [5] the authors adopt $\mathcal{M}=8.187957392$ and the stable fixed point is $Z_{c}=1.693848849$. The linerarized system of $(9)$ near the point $\left(Z_{s}, 0\right)$ is a harmonic oscillator:

$$
\ddot{Z}+V_{I n v}^{\prime \prime}\left(Z_{s}\right) Z=0,
$$

where

$$
V_{\text {Inv }}=-\mathcal{M} f_{0}^{\prime}(Z)+Z .
$$

Thus, in the invariant region, $Z$ oscillates with the frequency

$$
\sqrt{-\mathcal{M} f_{0}^{\prime \prime \prime}\left(Z_{s}\right)}
$$

A similar kind of linear equation was found by [3].

\section{Numerical analysis}

To integrate the system (6), we fix the parameters $A$ and $C$ as in [5], namely

$$
A=0.089, \quad C=0.139 .
$$

These values correspond to physical measurements, the effective radius being $a=34.7 \mathrm{~mm}$. The value of $a$ determines the inertial momenta of the spinning top.

We adopt the value $\mathcal{M}=8.188$ for which the two equilibrium points of the linearized system are equal.

The numerical method we have chosen to cope with the integration in regions of phase space where the system can become stiff is a Runge-Kutta-Fehlberg 7-8.

The Eqs. (6e), (6f) and (6j) are singular whenever $\theta=0$ or $\pi$. To overcome this issue we control the numerators of such expressions. For instance, in the case of Eq. (6e) we fix to zero the entry of the field corresponding to $\psi$ whenever it is

$$
\left|p_{\psi}-p_{\theta} \cos \theta\right| \leq \text { tol } .
$$

The same control is kept in the other two equations. The value of tol is calibrated numerically, not having found any significant difference for values lesser than 0.0001 . We verify that to have a stable flight of the spinning top $p_{\phi}$ and $p_{\psi}$ should be close; also in this case our control makes sense. Let us notice that the obtained results confirm the ones in [2].

Finally, the initial conditions have been picked up close to the equilibrium point $Z_{c}$ associated to $\mathcal{M}=8.188$. 


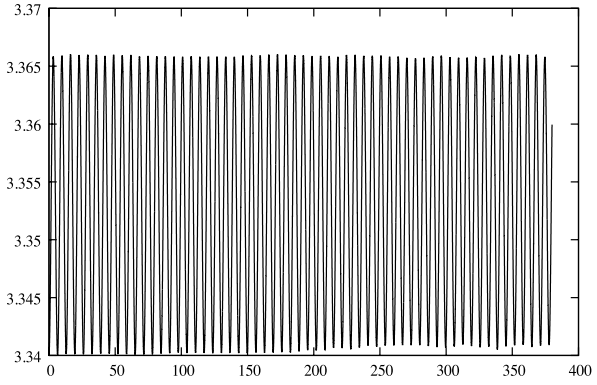

(a)

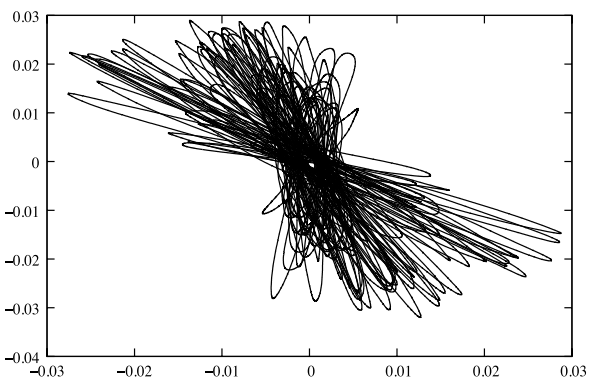

(b)

Fig. 2. (a) Time evolution of $z$-coordinate; (b) $(x, y)$-coordinates.

\subsection{Non symmetric solution}

In [5], the authors propose a symmetric solution of the system with small precession, explicitly

$$
\begin{aligned}
& \mathbf{q}=(r \cos \Omega t, r \sin \Omega t, h, \alpha, \Omega t, w t)^{T} \\
& \mathbf{p}=\left[-r \Omega \sin \Omega t, r \Omega \cos \Omega t, 0,0, \Omega\left(A \sin ^{2} \alpha+C \cos ^{2} \alpha\right)+C w \cos \alpha, C(w+\Omega \alpha)\right]^{T},
\end{aligned}
$$

where the spinning velocity $w$ can be fixed freely and then we can find the values of $r, \alpha, h$ and $\Omega$.

In the case where $p_{\phi}$ and $p_{\psi}$ are different but very close to each other, we take the same initial conditions as in the symmetric case except for $p_{\psi}=5.0001$, and the remaining initial conditions are $x=y=\phi=\psi=p_{x}=p_{y}=p_{z}=p_{\theta}=0, z=Z_{s}$, $p_{\phi}=5.0$ and $\theta=0.005$. The results are displayed in Figs. 2(a) and 2(b). Let us notice that the coordinates $(x, y)$ are no longer fixed at the origin, nevertheless the spinning top remains stable for about the same extent of time as in the symmetric case. From now on we will work with these initial conditions for the angular velocities $p_{\phi}$ and $p_{\psi}$. The fligth time of our numerical computations overcome the 900 units of times.

\subsection{Dissipative equations}

The dissipation terms come from two different types of motion involved in the flight of the spinning top: translation and rotation. Therefore two positive constants are needed, namely, $C_{T}$ and $C_{R}$. The dissipative terms added to the Eqs. $(6 \mathrm{~g})-(6 \mathrm{i})$ are $-C_{T} p_{x},-C_{T} p_{y}$ and $-C_{T} p_{z}$ respectively. Since the rotational velocity is very high compared with the translational velocity, we will model this friction with a quadratic term. Therefore the dissipative terms added to the Eqs. $(6 \mathrm{j})-(6 \mathrm{l})$ are $-C_{R} p_{\theta}\left|p_{\theta}\right|$, $-C_{R} p_{\psi}\left|p_{\psi}\right|$ and $-C_{R} p_{\phi}\left|p_{\phi}\right|$ respectively. The absolute value is used in order to preserve the sign of the rotation velocity ensuring that all three terms act against the rotation of the spinning top. The value of the dissipation constant has been obtained by numerical calibration. Numerical simulations using $C_{T}=C_{R}=0.1$ are displayed in Figs. 3(a) and 3(b). It can be observed that the spinning top falls after a short period of time. Figure $3(\mathrm{~b})$ shows how the stable position in the $(x, y)$-plane is lost.

The $z$-coordinate could have a bigger variation than that of the $x$ or $y$-coordinates, and then a different third dissipative constant could be introduced to add more dissipation in the vertical direction. Numerical simulations have been carried out using a third dissipation constant for the $z$-coordinate, finding that even more longer periods of time of stable flight of the spinning top are feasible. 


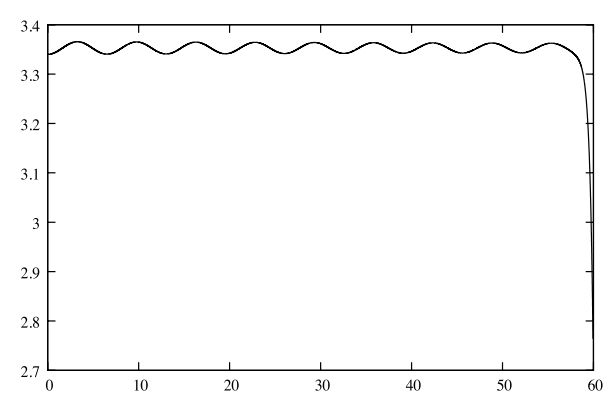

(a)

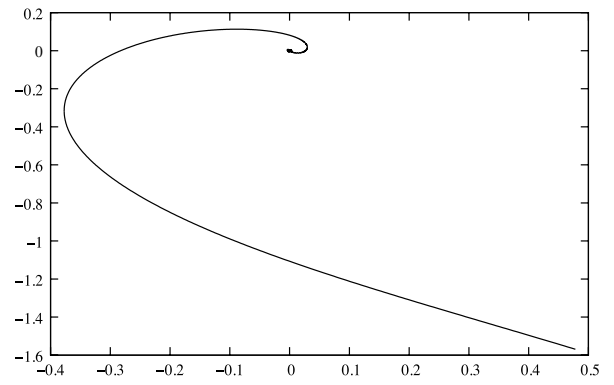

(b)

Fig. 3. (a) Time evolution of $z$-coordinate; (b) $(x, y)$-coordinates.

\subsection{Forced equations}

There are different strategies to force the equations of motion; they are basically of two types: electromagnetic and mechanical. Those using electromagnetic fields, which introduce torque forces into the system, have been extensively implemented in the past (see [6]). In the present effort instead, we aim to study mechanical forcing schemes, in particular, the forcing of the vertical coordinate of the position of the permanent magnetic base by small motions simulated in order to inject energy into the system.

Taking the $Z$-coordinate from the initial condition $Z_{c}$, we add one term to the equilibrium point $Z_{s}$, i.e. we perform the following change

$$
Z_{s} \longmapsto Z_{s}(1+\beta \cos \omega t),
$$

and refer to this perturbation as a parametric forcing.

We will analyze, locally, the effects of a parametric perturbation of the magnetic base in the $Z$ coordinate, which will lead us to a Mathieu equation. Afterwards, we will add a dissipative term and obtain a stability diagram with an Arnold's tongue structure (see [10]).

To model a parametric perturbation of the Levitron's magnetic base, we change the equilibrium point of the system (12) by performing the transformation (14). Therefore, the linearized equation near the point $\left(0, Z_{s}\right)$ reads

$$
\ddot{Z}+V_{\text {Inv }}^{\prime \prime}\left(Z_{s}(1+\beta \cos (\omega t))\right) Z=0 .
$$

where, from (13),

$$
V_{I n v}^{\prime \prime}=-\mathcal{M} f_{3}(Z)=-\mathcal{M} f_{0}^{\prime \prime \prime}(Z)=-\mathcal{M} \frac{90 Z^{2}\left(1+Z^{2}\right)-9\left(1+Z^{2}\right)^{2}-105 Z^{4}}{\left(1+Z^{2}\right)^{9 / 2}} .
$$

The parametric oscillation of the base is locally described by a Mathieu equation in the Z-coordinate, (15). Therefrom, we expect that the parameters $\beta$ and $w$ give rise to a stability diagram for the the Levitron's solutions with an Arnold's tongue structure.

When introducing a dissipative term to the equation we expect that the perturbation added to the magnetic base, with a suitable calibration of the amplitude and frequency, will inject energy into the system leading to a steady levitation of the spinning top for a rather long time. The parameters $\beta$ and $w$ will be found numerically since the local theoretical analysis could turn out very complicated. 
The equations of motion (6) plus the dissipative terms introduced in Sect. 3.2 provide a realistic model for the flight of the spinning top. In this section we use the forced equations as a way to simulate a small vertical motion of the magnetic base to inject energy into the system. Numerically, we will show that this results in a long period stable flight of the Levitron.

\section{Stability of the Levitron solutions}

We are interested in studying the non-linear stability of any solution of Eq. (6). A standard method to gain this information consists in computing the well-known maximum Lyapunov characteristic exponent (MLCE) for each orbit. For our study, it is important to use a fast method to compute the MLCE, since the variation of the flying time is very large when we change the value either of the parameters or the initial conditions of Eq. (6). Let us state that by flying time we refer to the period while the spinning top levitates in a bounded region around the fixed point. A novel method to determine the MLCE fast enough is the Mean Exponential Growth factor of Nearby Orbits (MEGNO). In this section we outline this technique but a detailed description of the MEGNO can be found for instance in [11].

Let us recall that the MEGNO is a fast indicator of the dynamics which is efficient to examine both regular and chaotic components of the phase space of a given dynamical system. Furthermore, it succeeds in revealing both the resonance structure and the location of stable and unstable periodic orbits. But herein, what interest us the most is that it provides a measure of hyperbolicity in chaotic domains which coincides with that given by the MLCE, but in a considerably shorter evolution time as already stated.

Consider the following equations of motion defined in $B^{\prime} \subseteq M_{h}$

$$
\dot{x}=\boldsymbol{v}(\boldsymbol{x}) \text {. }
$$

Now, let $\varphi(t)$ denote a solution of the flow (17) corresponding to a given initial condition $\boldsymbol{x}_{0}$, so that

$$
\varphi(t)=\left\{\boldsymbol{x}\left(t ; \boldsymbol{x}_{0}\right), \boldsymbol{x}_{0} \in B^{\prime}\right\} .
$$

Let us introduce the definition of the Mean Exponential Growth factor of Nearby Orbits (MEGNO), $Y(\varphi(t))$, given by

$$
Y(\varphi(t))=\frac{2}{t} \int_{0}^{t} \frac{\dot{\delta}(\varphi(s))}{\delta(\varphi(s))} s \mathrm{~d} s .
$$

where $\varphi(t)$ is the solution of the variational Eq. of (17)

$$
\dot{\boldsymbol{\delta}}=\Lambda(\varphi(t)) \boldsymbol{\delta}
$$

and $\Lambda(\varphi(t))$ is the Jacobian matrix of $(17)$.

The MEGNO's temporal evolution can be summarized in a single expression that provides its asymptotic behavior:

$$
\bar{Y}(\varphi(t)) \approx a_{\varphi} t+b_{\varphi}
$$

where $a_{\varphi}=\sigma_{\varphi} / 2$ and $b_{\varphi} \approx 0$ for chaotic motion, while $a_{\varphi}=0$ and $b_{\varphi} \approx 2$ for stable quasiperiodic motion. Departures from the value $b_{\varphi} \approx 2$ indicate that $\varphi$ is close to some periodic orbit, being $b_{\varphi} \lesssim 2$ and $b_{\varphi} \gtrsim 2$ for stable or near-unstable periodic orbits, respectively. 
Let us indicate that $\hat{\sigma}_{1} \equiv Y(\varphi(t)) / t$ verifies

$$
\hat{\sigma}_{1}\left(\varphi_{q}(t)\right) \approx \frac{2}{t}, \quad \hat{\sigma}_{1}\left(\varphi_{i}(t)\right) \approx \sigma_{i}, \quad t \rightarrow \infty,
$$

rendering clear that, for regular motion $\hat{\sigma}_{1}$ converges to 0 faster than $\sigma_{1}$, which vanishes at a rate $\ln t / t$, while for chaotic motion both quantities approach the positive MLCE at a similar rate.

\subsection{Bifurcation diagram}

We studied the flying time variation while changing the damping and perturbation parameters. We set the parameter $\mathcal{M}=32.75367$.

With that aim we adopted for the damping parameters the values $C_{T}=0.2$ and $C_{R}=0.01$ and computed the flying time for different values of $\beta$ and $\omega$. The set of values of $\beta$ taken for the simulations are

$$
\beta=n(0.001) \quad n=0, \ldots, 2000,
$$

and for each of these values of $\beta$, we took the values of the oscillation frequency

$$
\omega=n(0.004) \quad n=0, \ldots, 1000,
$$

which accounts for a total of two millons integrations. The initial conditions are the same as those used in Sect. 3.1. The initial step of integration is 0.001 and the approximation error is of the order of $10^{-14}$. The stop condition used for the numerical integration is

$$
|X|+|Y|+\left|Z-Z_{s}\right|>2
$$

where $Z_{s}$ is the equilibrium point found in previous sections. All the simulations were performed by the GPU-cluster of IIMAS-UNAM.

The results of these simulations are shown in Fig. 4. The vertical axis corresponds to values of $\beta$ and the horizontal one to values of $\omega$. Every point in the graph is coloured according to the value of the flight time in a logarithm scale. The largest flight time is $96 u$.

The plot 4 looks similar to the structure of Arnold's tongues that we find in the bifurcation diagram of the nonlinear Mathieu equation. The solutions with exponential growth and exponential damping have a short flying time since such solutions leave the equilibrium region in a short time and the top falls to the ground; at this point the integration has to stop. On the other hand, solutions with large flying times correspond to the cases in which a delicate equilibrium is preserved between the excitation and dissipatives forces with the nonlinear behavior of Eq. (6). Figure 4 shows that the accumulation of points with larger flying time are located in the region given by $0<\omega<1.0$ and $0<\beta<2.0$.

The parametric excitation can not restore the loss of the rotational energy. Nevertheless, the stable regions in the Arnold's tongues correspond to solutions which avoid the growth of the absolute value of the following terms

$$
\frac{p_{\psi}-p_{\phi} \cos \theta}{\sin ^{2} \theta} \text { and } \frac{\left(p_{\phi} \cos \theta-p_{\psi}\right)\left(p_{\psi} \cos \theta-p_{\phi}\right)}{\sin ^{3} \theta},
$$

when $\theta$ is close to zero. The values of $\left|p_{\phi}\right|$ and $\left|p_{\psi}\right|$ reduce their value in a monotonic fashion while the magnitude of (24) remains bounded in the stable regions on Fig. 4. 


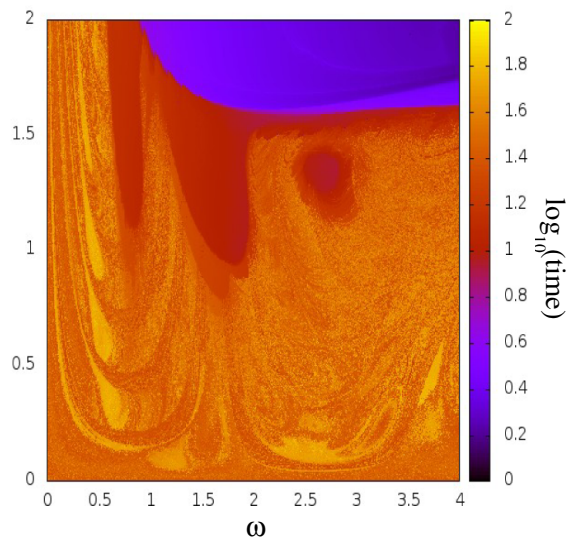

(a)

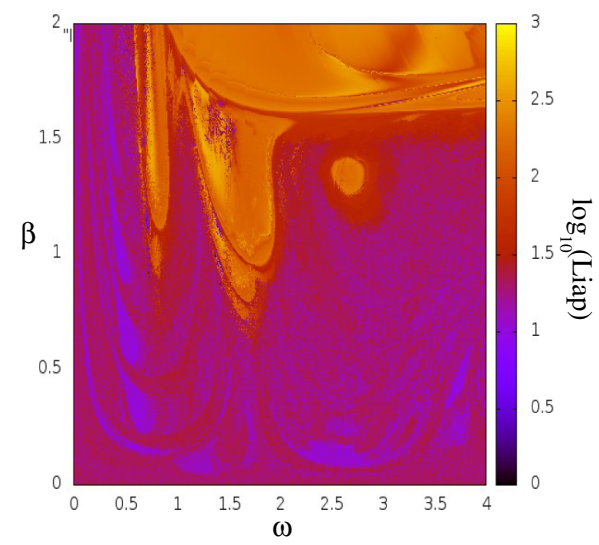

(b)

Fig. 4. (a) The axes correspond to the perturbation frequency $(\omega)$ and the perturbation amplitude $(\beta)$, a) flight time is represented by colour intensity in a logarithmic scale; b) The logarithm of largest Lyapunov exponent is represented by colour intensity

We would like to count with a method to estimate the Levitron's flying time without having to perform the whole numerical integration until the top falls to the ground. To this end, we computed the largest Lyapunov exponent for our numerical simulations by means of the MEGNO method described in the previous section. We computed a reduced set of the variational Eq. (20). Indeed, in this case we only considered the linear equations of the position and angular variables. The reason to discard the variational equations corresponding to the momentum variables in (20) is due to the behavior of the Eq. (24). The full system (20) is very unstable when $\theta \rightarrow 0$ since the matrix $\Lambda(\varphi(t))$ becomes singular in such a case.

We computed the bifurcation diagram of the largest Lyapunov exponent in a similar way to that used for Fig. 4, but in this case the colour code corresponds to the value of the largest Lyapunov exponent. The outcome bifurcation diagram is shown in Fig. 4b), where the value of the largest Lyapunov exponent is given in an logarithmic scale. We observe that the set of Arnold's tongues of the largest flight time in Fig. 4 matches the corresponding Arnold's tongues in Fig. 4b). Therefrom, we can conjecture that the early computation in time of the Lyapunov exponent is a good indicator that permits to predict the Levitron's flight time.

\section{Conclusions}

In this paper we studied the dynamics of the Levitron, in particular we were interested in determining the flying time when we added dissipation in the equations of motion. In such a case the flying time is limitated to $t<23 u$. In order to extend this time, we introduced a mechanical perturbation to the magnetic field of the permanent magnet, which corresponds to a parametric forcing (14). We made a systematic study of the flying time as a function of two parameters of the perturbation, $\beta$ and $\omega$, and obtained a detailed bifurcation diagram which is shown in Fig. 4. In this diagram we can appreciate the formation of Arnold's tongues where the flying time exceeds the limit time $t<23 u$. We also analysed the stability of these solutions by means of a fast technique to compute the maximum Lyapunov exponent, the Mean Exponential Growth factor of Nearby Orbits (MEGNO). The results are displayed in Fig. 4(b) (where the stable zones correspond to values of the MEGNO 


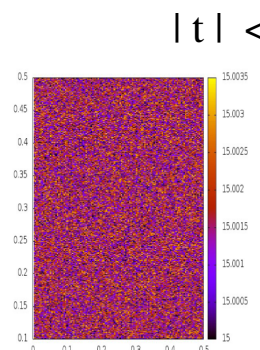

(a)

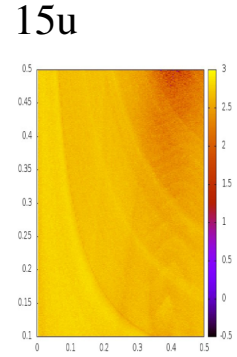

(b)

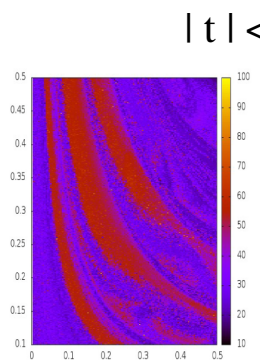

(c)

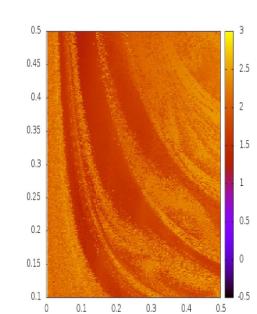

(d)

Fig. 5. Bifurcation diagrams of the parametric perturbation. $\omega$ is represented in the horizontal axes and $\beta$ in the vertical axes. Figures(a) and (c) show the bifurcation diagram of the flying time where colour intensity represents the flying time; Figs.(b) and (d) show the bifurcation diagram of MEGNO; there the colour intesity represents the MEGNO value. Figures(a) and (b) are limitated in time to $t<15 u$; for Figs.(c) and (d) the limit time is $t<100 u$

close to 2) which shows a very similar structure of Arnold's tongues as the one found in the flying time diagram.

In the end we concluded that the MEGNO serves as an early indicator of the stability of the solutions to the Levitron's equations. Figure 5 shows a small region of the bifurcation diagrams where the flying time was limited to $t<15 u$ (Fig. 5(a)). In this case we could not distinguish any structure similar to Fig. 4, instead the plot looks like a random distribution. However, when we increase the maximun flying time to $t<100 u$, we can appreciate the Arnold's tongues structure as can be seen in Fig. 5(c). On the other hand, in the bifurcation diagram of the Lyapunov exponent for $t<15 u$ given in Fig. 5(b), an early formation of the Arnold's tongues can be detected where MEGNO is close to the value 2. Figure 5(d) shows a clearer formation of the Arnold's tongues for the MLCE diagram. In sum we can state that MEGNO allows for an early indication of stability of any solution of the Levitron equations, and the stable solutions are good candidates to have very long flying times.

This work was founded by FENOMEC-UNAM and CONACyT project 133036-F. We also express our gratitude to Pablo Cincotta for his invaluable help in description of MEGNO routines and Ana Perez for her assistant in the computer implementation. Finally, we would also like to thank the anonymous referee whose valuable comments have improved the presentation of the paper.

\section{References}

1. S. Earnshaw, Trans. Cambridge Philos. Soc. 97 (1842)

2. M.V. Berry, Proc. R. Soc. London A. 452, 1207 (1996)

3. M.D. Simon, L.O. Heflinger, S.L. Ridgway, Amer. J. Phys. 65, 286 (1997)

4. H.R. Dullin, R.W. Easton, Phys. D. 126, 1 (1999)

5. R.F. Gans, T.B. Jones, M. Washizu, J. Phys. D: Appl. Phys. 31, 671, (1998)

6. A.T. Pérez, P. García-Sánchez, Amer. J. Phys. 83, 133 (2015)

7. J.D. Jackson, Classical Electrodynamics 2nd edn. (Wiley, 1975)

8. L. Meirovitch, Methods of Analytical Dynamics (Dover Publications, 2009) 
9. J. Geiser, K.F. Lüskow, R. Scheider, Dynamical Syst. 29, 208 (2014)

10. D.W. Jordan, P. Smith, Nonlinear Ordinary Differential Equations: An Introduction to Dynamical Systems (Oxford Texts in Applied and Engineering Mathematics). 3rd edn. (Oxford University Press, 1999)

11. P.M. Cincotta, C.M. Giordano, C. Simó, Phys. D. 182, 11 (2003)

AQ: The caption from Fig. 2 have been modified, since they have been repeated? Please check is this OK? 